\title{
Observation of the Annular Solar Eclipse of May 312003 in far ultraviolet spectral region
}

\author{
V. V. Katyushina, T. V. Kazachevskaya and A. A. Nusinov \\ Institute of Applied Geophysics, Russian Hydrometeorological Service, Moscow \\ email:geophys@hydromet.ru
}

Changes of extreme ultraviolet (EUV) Solar flux were registered during the Annular Solar eclipse of May 31 2003, when CORONAS-F satellite (Oraevsky et al., 2002) intersected thrice a zone of the Solar eclipse. During these periods the solar emission was measured in visible and extreme UV spectral regions (near $L_{\alpha}=121.6 \mathrm{~nm}$ by VUSS instrument). Records by VUSS ( $\sim 120 \mathrm{~nm}$ ) and the optical (visible) channel of EOS instruments on UTC scale for May 31, 2003 are given on Fig. 1.

One can see a sequence of dips in the signal. "Rectangular" dips correspond to an entrance of the satellite's orbit into the night part, while "triangle" dips correspond to signal reductions due to the eclipse. A visible channel is auxiliary, its records were shown only to illustrate eclipse effects. On May 31, 2003, an annular eclipse of the Sun could be visible from a broad corridor which traversed the far Northern Hemisphere.

Fig.2 demonstrates the map of eclipse's zones at the Earth's surface (Espenak \& Anderson, 2003). Parts of the satellite orbits corresponding to crosses of these zones are presented by thick lines as well as moments of maximal eclipse effect.

Mutual position for the Sun and the Moon was calculated as well as a parameter $X$ a part of the Solar disc that was not shaded by the Moon as it had been seen from the satellite. There exists some peculiarities of eclipse observations on-board moving celestial body, such as an Earth's satellite. In this case the "open" part of the Sun, as it could be seen from the satellite, changed not only its value, but also changed rapidly its position on the Sun.

Observed eclipse effect maximum (ratio of a minimum signal, when the eclipse was registered, to a maximum signal without an eclipse) were $0.147,0.091$ and 0.762 at 03:05:39 UTC, 04:28:20 UTC and 05:53:20 UTC correspondingly. Minimum values of $X$ were $0.201,0.136$ and 0.743 correspondingly.

It can be seen from these data, that the instrument on-board the satellite could not observe a maximum annular eclipse phase $X=0.112$ for CORONAS-F, although as it can be seen on Fig. 2, at the time of the second flight the satellite crossed the Earth's surface region of the maximum phase of eclipse.

Figures 3a-3c represents changes of normalized VUSS signal (solid line) together with $X$ (dashed lines) for three sequential satellite passages of the eclipse band. On Figure 3a$3 \mathrm{c}$ one can see characteristic peculiarities that were revealed after comparison of these figures.

A relation between an open part of the Sun and a VUSS signal changed from one turn to another. At the 1-st and 2-nd turns minimum VUSS signal values were smaller that of $X$. But when $X>\sim 0.7$ and at the 3 -d turn there is reverse relation. It may be interpreted as a result of two effects: spatial distribution of different emitting elements and a limb darkening. Indeed, there were three active regions $(365,368$, and 373$)$ at the visible Solar disk during the eclipse period. Their moving relative to the Moon shadow, as seen from CORONAS-F satellite could cause small, but distinctive UV variations different from those ones in visible light. 


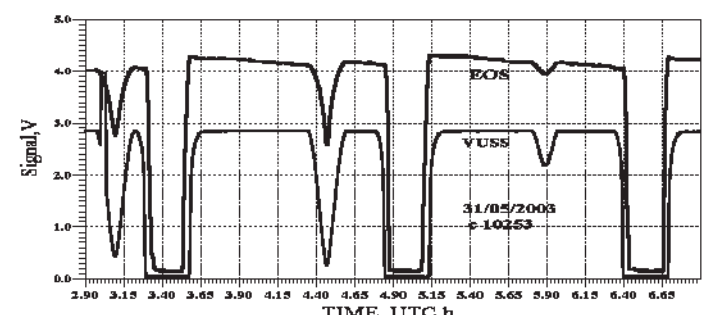

TIME, UTC h

Figure 1. VUSS optical and EUV channel signals

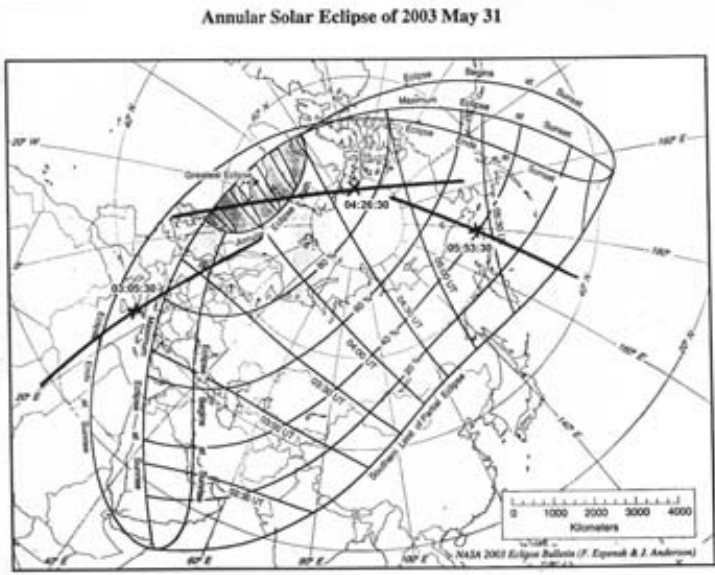

Figure 2. Crossings of the eclipse's zones by the sequential CORONAS orbits
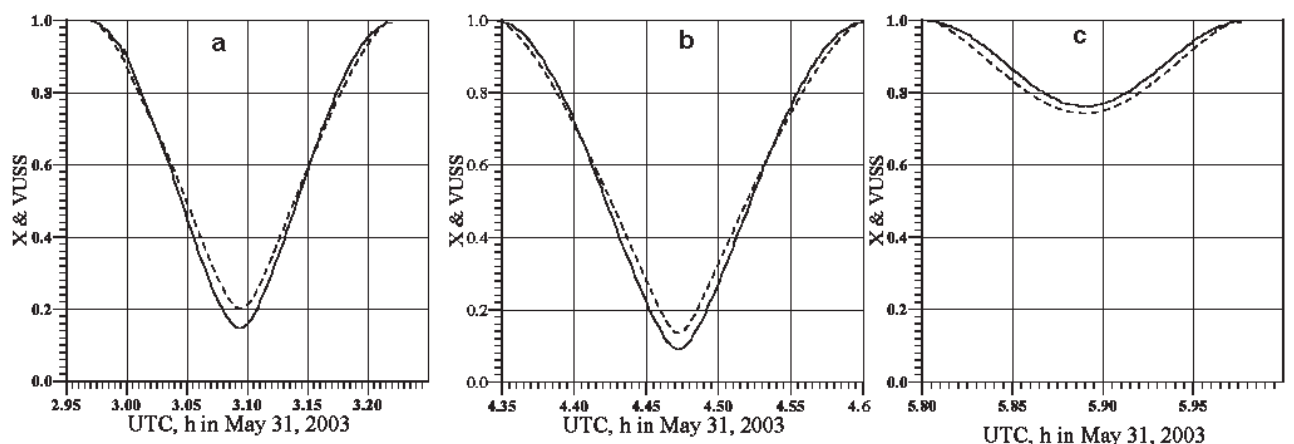

Figure 3. Normalazed EUV and calculated optical signal $X$ for three CORONAS turns

\section{Acknowledgements}

Authors are grateful to K.V.Kuimov and V.V.Chazov (SAI of the MSU) for the calculations they performed. The work was supported by the RFBR (project No. 03-02-17109).

\section{References}

Espenak F., Anderson J 2003 NASA 2003 Eclipse Bulletin, 12.

Oraevsky V.N., Sobelman I.I, Zhitnik I.A. \& Kuznetsov V.D. 2002 Uspekhi Fizicheskikh Nauk 45, 886-896. 